\title{
The Role of Rehabilitation in Cancer Treatment
}

Jennifer C. Smith

jccarter32@yahoo.com

Follow this and additional works at: https://nsuworks.nova.edu/ijahsp

Part of the Medicine and Health Sciences Commons

\section{Recommended Citation}

Smith JC. The Role of Rehabilitation in Cancer Treatment. The Internet Journal of Allied Health Sciences and Practice. 2016 Jan 01;14(2), Article 8.

This Manuscript is brought to you for free and open access by the College of Health Care Sciences at NSUWorks. It has been accepted for inclusion in Internet Journal of Allied Health Sciences and Practice by an authorized editor of NSUWorks. For more information, please contact nsuworks@nova.edu. 


\section{The Role of Rehabilitation in Cancer Treatment}

\section{Author Bio(s)}

Jennifer C. Smith, OTD, MBA, OTR/L is Director of Operations at Eastern Physical Medicine and Rehabilitation, LLC in Greenville, NC. She is also a licensed occupational therapist in North Carolina. Her interests include chronic pain management, aging in place and rehabilitation needs of cancer patients 


\title{
IJAHSP \\ The Internet Joư⿱n一⿰口乚一al of Allied Health Sciences and Practice
}

Dedicated to allied health professional practice and education

Vol. 14 No. 2 ISSN 1540-580X

\section{The Role of Rehabilitation in Cancer Treatment}

\author{
Jennifer C. Smith, OTD, MBA, OTR/L \\ Eastern Physical Medicine and Rehabilitation, LLC \\ United States
}

\begin{abstract}
In the past, persons diagnosed with cancer were thought to need to prepare for death. Currently, the five-year survival rate for all cancers has increased to $66.2 \%$ from 2005 to 2011 as compared to $49 \%$ from 1975 to $1977 .{ }^{1}$ This increased survival rate can be attributed to earlier diagnosis, improved treatments, and the supportive care provided today. Cancer has become one of the most disabling and costly conditions affecting Americans today. ${ }^{2}$ A prevalent cause of disability and emotional distress in cancer survivors is physical dysfunction, and cancer survivors frequently report many unmet needs. ${ }^{3}$ This information suggests potential opportunities for rehabilitation professionals to help meet the needs of cancer patients. Rehabilitation for cancer patients may occur along the continuum of care including acute care services, inpatient rehabilitation facilities, skilled nursing facilities, home- health and outpatient therapy services. This editorial describes how rehabilitation professionals could become involved in the care of patients dealing with a diagnosis of cancer.
\end{abstract}

\section{Introduction}

Cancer is one of the most widespread, disabling, and expensive health care conditions affecting people in the U.S today. Today, the five-year survival rate for all cancers has increased to $66.2 \%$ from 2005 to 2011 as compared to $49 \%$ from 1975 to $1977 .{ }^{1}$ More than $40 \%$ of persons born today will develop some type of cancer during their lifetime. ${ }^{2}$ Persons diagnosed with cancer are surviving longer due to early detection and remarkable improvements in treatment. ${ }^{5}$ Despite the positive effects of treatment, most cancer survivors do not fully return to their previous state of well-being. Cancer survivors may suffer from a wide range of functional problems ranging from fatigue, decreased physical fitness, and psychological problems to specific problems related to the type of cancer such as speech impairment and swallowing difficulty after head and neck cancer. ${ }^{6}$

While cancer treatment adds years to the lives of patients, rehabilitation services can add much quality to life by addressing the impairments caused by cancer. Physical therapy, occupational therapy, and speech language pathologists can address the individual needs of patients based upon a thorough evaluation and treatment plan. The patient age, cancer pathology, anticipated progression of the disease, and associated treatments should be considered when determining appropriateness of rehabilitation. Some of the physical and functional problems following cancer treatment include pain, weakness, impaired performance of activities of daily living, fatigue, and cognitive deficits. ${ }^{5}$ The specialty of cancer rehabilitation seeks to reduce and, when possible, eliminate functional losses associated with cancer and its treatment. ${ }^{7}$

\section{Cancer Care Today}

The American Cancer Society (ACS) in 2015 reports approximately 14.5 million Americans are alive today with a history of cancer. ${ }^{4}$ Some of these persons are actively receiving treatment, while many others were diagnosed years ago and are currently cancerfree. Nearly every person in the US today has a friend or family member that has been directly affected by cancer. The ACS predicts that nearly 1,658,370 new cancer cases will be identified this year and 549,430 Americans will die of cancer during 2015 . This is equal to about 1,620 deaths per day. In the United States, cancer is the second most common cause of death and is surpassed only by heart disease. While cancer affects persons at all ages and stages of life, cancer is more frequently diagnosed in older persons due to $78 \%$ of all cancer diagnoses are in persons aged 55 or older. ${ }^{4}$ Also, people who smoke, eat an unhealthy diet, or are physically inactive have a higher risk of cancer. The costs of cancer care are staggering in the US. The Agency for

(c) The Internet Journal of Allied Health Sciences and Practice, 2016 
Healthcare Research and Quality (AHRQ) estimates that the direct medical costs (total of all health care expenditures) for cancer in 2011 were $\$ 88.7$ billion. ${ }^{4}$

Many improvements in the diagnosis and treatment of cancer have increased survivorship, therefore cancer has become a chronic condition that requires long-term management, including rehabilitation. ${ }^{8}$ Cancer patients have many different physical, emotional, social, financial, and spiritual needs that require help from an integrated team of oncologists, physiatrists, palliative professionals, rehabilitation professionals, nurses, chaplains, social workers, and case managers. ${ }^{9}$ Although the diagnoses and clinical presentations of cancer patients vary greatly, many of these persons have one thing in common: they experience a loss of function that severely affects quality of life. A study comparing health-related quality of life (HRQOL) in cancer survivors with that of other persons found that cancer survivors reported a much worse HRQOL for both physical and emotional health compared to others. The HRQOL was more often influenced by physical issues than emotional issues. A leading cause of emotional distress in cancer survivors was physical disability. ${ }^{2}$

\section{The Need for Rehabilitation}

Physical disability can be addressed through rehabilitation services provided in acute care, inpatient rehabilitation facilities, skilled nursing facilities, home-health, and outpatient settings. Rehabilitation provides persons with disabilities the tools they need to attain independence and self-determination. This is especially important in the case of cancer, since rehabilitation can improve the quality of life for patients with cancer and their families. Based upon the biopsychosocial model and a holistic approach of rehabilitation, a cancer rehabilitation team encompasses multidisciplinary efforts including medical, psychological, physical therapy, occupational therapy, speech language pathologist, rehabilitation nursing, and recreational therapy depending upon the functional status of the patient. $^{3}$

Since oncology practice is frequently focused on observation, treatment, and reduction of symptoms, functional problems are less likely to be addressed. Some of the physical and functional problems associated with cancer and its treatment include pain, weakness, impaired performance of activities of daily living, fatigue, and cognitive deficits. ${ }^{5}$ In 2008 , a questionnaire about cancerrelated symptoms and functional problems was completed by 202 patients receiving outpatient cancer treatment. ${ }^{10}$ The questionnaire indicated that $72 \%$ of all patients had symptom-related complaints and $57.9 \%$ of those complaints indicated cancerrelated fatigue. Functional problems were reported by $65.8 \%$ of the patients, most of which were ADL difficulty. Difficulty with ambulation was reported by $22.8 \%$ of patients, balance problems were reported by $17.8 \%$ of patients, and needing support to walk was reported by $13 \%$ of patients. Almost one in five persons (18.1\%) reported cognitive problems. ${ }^{10}$ Each of these functional problems are appropriate for rehabilitation intervention.

Cancer patients in all phases of treatment may benefit from a rehabilitation plan. The physiatrist can assess the functional needs of the patient and develop an overall therapy plan, along with the oncology care, comorbidities, and psychosocial challenges. ${ }^{9}$ The purpose of inpatient cancer rehabilitation is to restore or maximize the functional status of patients with cancer. ${ }^{9} \mathrm{~A}$ retrospective record review of 153 patients discharged from an oncology rehabilitation unit from 2008-2010 provided descriptive data of the patients. The ages of patients varied widely from 38 to 97 years old, over half $(60 \%)$ of the patients were women, and $40 \%$ lived alone prior to admission. The most common diagnoses were colorectal cancer (29\%), metastatic disease (16\%), and urinary organ cancer ( $9 \%$ ). The goals of therapy were met for $77 \%$ of the patients and average functional independence measure (FIM) gain was 17.1 points. Lower FIM gain was associated with older age and a diagnosis of uterine or brain cancer. ${ }^{8} \mathrm{~A}$ systematic review of literature on the effectiveness of multidimensional cancer survivor rehabilitation programs and the cost-effectiveness of cancer rehabilitation found acceptable cost-effectiveness ratios or cost savings for interventions that produced significant health gains. ${ }^{6}$

There is a need to better understand and clarify the field of cancer rehabilitation, including determining appropriate screening for impairments and subsequent disability as well as identification of health care professionals who are qualified to treat the unique problems of cancer patients. Rehabilitation professionals are focused on functional outcomes including activities of daily living and instrumental activities of daily living. It is important to screen cancer patients for physical impairment which may range from mild to severe. The problems must be identified and treated in order to improve the physical and psychological outcomes of cancer survivors. Impairments and disability are related but not the same. Mild impairments can cause significant disability while some severe impairments may only cause minor disability. This leads to the importance of proper screening for impairments and determination of how the impairments relate to function. ${ }^{2}$

The four areas of cancer rehabilitation intervention that can address the scope and course of the illness include preventive, restorative, supportive, and palliative. The oncology and rehabilitation teams should work together to determine the stage of the continuum of care on which to focus the client's treatment. Preventive interventions are started early either before or immediately after treatment. This treatment is intended to lessen the impact of expected disabilities, emphasizing patient education and

(C) The Internet Journal of Allied Health Sciences and Practice, 2016 
approaches to improve physical function and general health status. Psychological counseling can address early issues related to adjustment and allow for prompt intervention. Restorative interventions are focused upon those patients that are cured or in remission but have a residual physical impairment and disability. The goal is to maximize and possibly return to previous levels of physical, psychological, social, and vocational functioning. Supportive rehabilitation involves attempts to improve the self-care skills and mobility of the current cancer patient. These efforts may include prosthetic training, instruction in use of other devices for mobility or self-care, emotional support, and adjustment to disability. Palliative care interventions are provided when the advanced disease progresses and the disability is increased. The interventions and goals center on increasing and maintaining comfort and function of patients with terminal illness. Some palliative care treatment goals include pain control, prevention of contractures and pressure ulcers, prevention of unnecessary deterioration from inactivity, and psychological support for patient and caregivers.

A rehabilitation team may include a physical therapist, occupational therapist, and speech language pathologist in addition to many other professionals. The national associations for each of the rehabilitation disciplines provide literature to assist therapists in planning treatment for cancer patients. The Oncology Section of the American Physical Therapy Association (APTA) provides fact sheets for the treatment of radiation fibrosis, lymphedema, head and neck cancer, and chemotherapy-induced peripheral neuropathy. ${ }^{11}$ The American Occupational Therapy Association (AOTA) has generated a fact sheet that describes occupational therapy intervention in cancer care in order for therapists to facilitate optimal performance in daily activities both from a physical and psychological perspective. ${ }^{12}$ The American Speech-Language-Hearing Association (ASHA) provides in-depth information on therapy for swallowing problems that can occur with head and neck cancers. ${ }^{13}$ There are a variety of functional deficits that can be treated by the interdisciplinary team. Rehabilitation professionals would benefit from training in the area of oncology rehabilitation in order to provide the most effective treatment for patients.

"Whether individuals are actively receiving treatment for a cancer diagnosis or are focused on rebuilding their lives after completion of treatment, clients with cancer experience altered life roles and occupations."14 Therapy practitioners have the potential to play a major role in the rehabilitation of clients with cancer, particularly in the acute care setting. One of the most challenging aspects of providing therapy to clients with cancer is setting realistic and appropriate goals, and determining which areas of occupation to address first. The best way to deal with this process is to involve the client and family in the goal setting process, and to guide the client toward realistic short and long term goals that are achievable and safe. ${ }^{14}$

\section{Cancer-Related Impairments}

Cancer patients must learn coping skills for chronic health conditions and treatment. Learning to effectively deal with hair loss, neuropathy from chemotherapy agents, fatigue, and anorexia related to chemotherapy and radiation therapy, as well as depression and anxiety related to the diagnosis and treatment of cancer is an important part of rehabilitation and recovery. ${ }^{15}$ Cancer patients must also cope with further psychological issues including family role reversal, employment issues, and insurance issues. Many persons with chronic health conditions must deal with such conditions as depression and fatigue, while many cancer patients experience unique impairments related to cancer treatment such as hair loss.

Identifying and addressing treatment-induced physical impairments that can lead to considerable disability is an important part of cancer care. ${ }^{2}$ Pain is one of the most common issues that cancer rehabilitation health care professionals routinely address. Pain can often be chronic and debilitating for cancer patients and survivors. Post-mastectomy, post-amputation, and post-thoracotomy pain syndromes have all been described in literature. While pain is common among cancer patients, many cancer patients may have pain due to musculoskeletal conditions that are not directly related to the cancer. A thorough evaluation to determine to cause of the pain can help to determine the most appropriate treatment. Deconditioning due to inactivity during cancer treatment can lead to other musculoskeletal conditions which can be treated with physical therapy. ${ }^{5}$ Inactivity and deconditioning can also contribute to cancer-related fatigue.

Cancer-related fatigue (CRF) is defined as "a distressing, persistent, subjective sense of physical, emotional, and/or cognitive tiredness or exhaustion related to cancer or cancer treatment that is not proportional to recent activity and interferes with functioning". ${ }^{16}$ The incidence of CRF in clinical trials ranges from $30 \%$ to $90 \%$, depending on cancer type and treatments. Some of the most important factors that contribute to CRF are treatment with cytotoxic chemotherapy, radiation therapy, anemia, pain, emotional distress, sleep disturbance, and poor nutrition. ${ }^{5}$ In addition to having a negative impact on quality of life and daily activities, fatigue is associated with financial loss and social isolation due to an inability to work following illness. ${ }^{17}$ Medical and pharmacological interventions can help to improve fatigue. Improving sleep, treating chronic pain, and treating depression have been shown to improve symptoms. Other rehabilitation interventions may include education on energy conservation and activity adaptation, low grade exercise program, adaptive devices, and cognitive-behavioral training. ${ }^{5}$ Exercise has a powerful effect on cancer-related fatigue. Multiple studies have shown fatigue levels were $40 \%$ to $50 \%$ lower in exercising participants including studies with small sample sizes. ${ }^{18}$

(C) The Internet Journal of Allied Health Sciences and Practice, 2016 
Chemotherapy-induced peripheral neuropathy is a possible side effect of chemotherapy and is one of the most prevalent neurologic complications of cancer treatment. It is estimated that peripheral neuropathy may develop in $50 \%$ to $60 \%$ of patients treated with taxanes and can also result from paraneoplastic phenomena as well. ${ }^{5}$ The symptoms can be subtle and include functional loss in sensation, proprioception, and motor function. Treatment can include balance training, visual compensation training, orthotics, equipment use, and patient education.

Steroid induced myopathy is thought to be secondary to the catabolic effect of steroids on the muscles. This can occur at the start of steroid therapy or when a dose is increased for a person on chronic maintenance therapy. This type of myopathy presents with subacute onset of weakness with muscle wasting, although generally no myalgia or muscle tenderness. Muscle enzymes and electromyography findings are normal in steroid myopathy and the diagnosis in determined by history and exclusion of other causes. Lower extremity weakness has a significant impact on ability to perform activities of daily living. ${ }^{5}$

Chemo-brain refers to mild cognitive impairment that can occur during or following chemotherapy. There are various possible mechanisms that may contribute to chemo-brain including vascular injury, oxidative damage, inflammation, injury to neurons, autoimmune responses, and chemotherapy-induced anemia ${ }^{1}$. Other possible causes of cognitive impairment include the effects of radiation therapy, narcotic medications, or cerebral metastases. Some of the symptoms of chemo-brain include difficulty with memory, attention, and organizational skills. The use of physical and occupational therapy to address pain management can lead to reduced opioid use and improvement in cognition. Speech and occupational therapy can further address cognition through regular therapy sessions, home programs, and memory aides. ${ }^{5}$ Cognitive dysfunction can also occur with the treatment of brain tumors.

Persons with brain tumors have been found in some studies to have shorter lengths of stay on acute rehabilitation units compared with other noncancerous brain disorders, possibly due to higher levels of functional independence on admission, fewer behavioral issues, better social support, and expedited discharge planning due to cancer-related prognostic factors. Most persons with brain tumors have multiple impairments depending upon tumor size and location, and the amount of tissue excised for patients that have undergone surgery. ${ }^{2}$ In a 2013 study by Khan and Amatya of 106 patients with primary brain tumor in an Australian community, the following impairments were reported: pain $(56 \%)$, head1aches $(42 \%)$, ataxia $(44 \%)$, seizures $(43 \%)$, paresis $(37 \%)$, cognitive dysfunction (36\%), visual impairment (35\%), and depression (20\%). ${ }^{19}$ Rehabilitation after surgery can improve functional outcome, justifying the delivery of rehabilitation services, even during the acute phase, to brain tumor patients, regardless of tumor type. ${ }^{20}$ Early mobilization of patients with brain-tumor related disability includes transfers from bed to wheelchair, ambulation, and wheelchair mobility.

Cancer-related spinal cord injury may represent the most common type of non-traumatic spinal cord injury. ${ }^{21}$ Symptoms can include weakness, autonomic dysfunction, sensory loss, and pain. Medical complications associated with recent $\mathrm{SCl}$ are common and can be life-threatening. Positive results have been demonstrated following rehabilitation of individuals with disability from spinal cord tumors, with significant functional gains measured after inpatient rehabilitation. ${ }^{2}$ Comprehensive rehabilitation care of $\mathrm{SCl}$ includes management of pain, autonomic dysreflexia, pulmonary and urinary tract infections, thromboembolic disease, bowel and bladder dysfunction, decubitus ulcers, limb contractures, and spasticity.

A study by Ruff et al was conducted to determine whether a directed rehabilitation program had an effect on survival, pain, depression, independence, and life satisfaction for Veterans who were non-ambulatory after treatment for spinal epidural metastasis. ${ }^{22}$ This study compared 12 consecutive Veterans with paraplegia who were admitted to an inpatient rehabilitation facility for 2 weeks of directed rehabilitation with a historical control group of 30 paraplegic Veterans who did not receive rehabilitation. The rehabilitation program consisted of transfer training, bowel and bladder care, incentive spirometry, nutrition, and skin care. The outcome measures included survival, independence, pain level, depression, and life satisfaction. The patients who received rehabilitation demonstrated longer median survivals, fewer deaths from spinal cord injury-related complications, less pain, lower depression scores, and higher satisfaction with life scores. Eight of the patients who attended rehabilitation were able to perform transfers independently (versus none in the control group) and nine returned home (versus six in the control group). ${ }^{22}$

Most skeletal cancer cases are of metastatic origin. Bony metastases are a frequent source of cancer-related physical impairment and require the active involvement of a rehabilitation team. Challenges do arise with the risk for fracture during mobility or therapeutic exercise, although the alternative of bed rest could contribute to debility and further functional loss. Learning to mobilize with weight bearing precautions and use of adaptive equipment is important for the patient.

A prospective study by Riesenberg and Lubbe was performed to determine changes in exercise capacity and quality of life before and after an interdisciplinary rehabilitation program for patients with lung cancer. ${ }^{23}$ This study included 45 patients with lung cancer

(C) The Internet Journal of Allied Health Sciences and Practice, 2016 
involved in a 28-day inpatient rehabilitation program that included standardized aerobic training. Functional status and healthrelated quality of life were evaluated on Day 1 and Day 28 of the study. A substantial increase was found in work performance using a bicycle ergometer and the 6-minute walk test. Also heart rate at rest was reduced and heart rate variability was increased. Furthermore, there was a significant improvement in quality of life and fatigue was reduced. This study concluded that a standardized aerobic endurance training program as a part of an inpatient oncological rehabilitation program for patients with lung cancer resulted in improvements in both physiological and psychological measurements after therapy. ${ }^{23}$

\section{Limitations of the Healthcare System}

Patients with a diagnosis of cancer are at risk of becoming survivors with lasting impairments across multiple body systems that could be addressed by rehabilitation professionals if appropriately identified. One of the most problematic barriers in cancer and rehabilitation is a lack of training in oncology rehabilitation for physiatrists as well as for other rehabilitation professionals including therapists and rehabilitation nurses. Without specific training dedicated to learning the nuances of oncology rehabilitation, it can be challenging to become skilled and effective providers. A further barrier can be limited communication between oncology and rehabilitation healthcare providers. This barrier can be overcome through dedicated partnerships between rehabilitation providers and oncologists. ${ }^{18}$

Limitations also occur within the healthcare system due to payer sources and federal regulations. The ability to admit all types of patients to the inpatient rehabilitation facility is limited by the federal regulations that require $60 \%$ of all patients admitted have one of 13 qualifying medical conditions that include: stroke, spinal cord injury, amputation, major multiple trauma, hip fracture, brain injury, neurological disorders (including multiple sclerosis, motor neuron disease, polyneuropathy, muscular dystrophy, and Parkinson's disease), burns and joint replacements (for patients with bilateral joint replacements, BMl greater than 50, or age 85 and older). ${ }^{24}$ These criteria change yearly based upon Congressional decisions. The specific medical conditions that qualify for inpatient rehabilitation services are becoming more specific and difficult to defend each year. Persons with a medical diagnosis that qualifies for an inpatient rehabilitation admission must also be able to meet the following criteria:

1. Require the active and ongoing interventions of multiple therapy disciplines.

2. Require active participation in an intensive rehabilitation therapy program that is generally defined as 3 hours of therapy, 5 days per week.

3. Require the close medical supervision by a physician that specializes in rehabilitation for medical conditions that require inpatient hospitalization.

4. Require an intensive and coordinated interdisciplinary approach to care in the IRF.

5. While the patient is not expected to achieve complete independence or return to prior level of functioning, it is expected that the patient will make functional improvements that are ongoing, sustainable, and of practical value. It is generally expected that a patient in the IRF will return home or a community-based environment. ${ }^{24}$

Because of the more stringent criteria for admission to an IRF, patients with cancer diagnoses may require more and more rehabilitation services to be performed in other locations such as the acute care hospital, skilled nursing facility, outpatient facility, or in the home with home health services. The ability to receive these services will also be dependent upon the payer source and further regulations. The rehabilitation and oncology teams must maintain an open relationship and close communication in order to determine the best rehabilitation plan for each patient.

\section{Conclusion}

By the year 2020, it is projected there will be 18 million cancer survivors in the United States. ${ }^{26}$ Nearly $80 \%$ of cancer patients have symptoms that would benefit from rehabilitation services, although fewer than $20 \%$ of those patients receive coordinated rehabilitation care. There are only a few dedicated oncology rehabilitation programs currently in the United States including Carolina's Rehabilitation and Baylor Institute for Rehabilitation. There are two fellowship programs for training physiatrists in cancer rehabilitation: Memorial Sloan-Kettering Cancer Center and University of Texas Anderson Cancer Center. ${ }^{27}$ There is certainly an opportunity to grow and develop oncology rehabilitation in many hospitals that have both a cancer care program and rehabilitation. Keys to a successful partnership for oncology and rehabilitation include cooperative education opportunities, strong communication, and consideration of alternate funding including grants and research studies.

Practitioners that work with cancer patients should review information on national association websites and journals. Awareness of the specific impairments that affect cancer patients is an important component of developing a rehabilitation plan. It is also critical to involve the full interdisciplinary team, the family, and caregivers, as well as input from the patient. The phase of cancer treatment, the goals of patient and caregivers, and the payer source should also be considered in determination a rehabilitation plan of care.

(c) The Internet Journal of Allied Health Sciences and Practice, 2016 


\section{REFERENCES}

1. National Cancer Institute. Table 1.5 Age-Adjusted SEER Incidence and U.S. Death Rates and 5-Year Relative Survival. Washington: National Cancer Institute, 2015 Retrieved February 10, 2016 from: http://seer.cancer.gov/csr/1975 2012/results merged/topic survival.pdf

2. Silver JK, Baima J, Mayer RS. Impairment driven cancer rehabilitation: An essential component of quality care and survivorship. CA: A Cancer Journal for Clinicians. 2013 Sep; 63(5):296-317. [PMID 23856764]

3. Ugolini D, Neri M, Cesario A, Bonassi S, Milazzo D, Bennati L, Lapenna LM, Pasqualetti P. Scientific production in cancer rehabilitation grows higher: a bibliometric analysis. Support Care Cancer. 2012 Aug; 20(8):1629-38. [PMID 21881910]

4. American Cancer Society. Cancer Facts \& Figures 2015. Atlanta: American Cancer Society, 2015. Retrieved January 6, 2016 from: http://www.cancer.org/acs/groups/content/@editorial/documents/ document/acspc-044552.pdf

5. Gupta AD, Lewis S, Shute R. Patients living with cancer: The role of rehabilitation. Australian Family Physician. 2010; 39(11):844-6. [PMID 21301657]

6. Mewes JC, Steuten LM, ljzerman MJ, Van Harten WH. Effectiveness of multidimensional cancer survivor rehabilitation and cost-effectiveness of cancer rehabilitation in general: A systematic review. The Oncologist. 2015; 17:1581-93. [PMID 22982580]

7. Franklin D, Delengowski AM, Yeo TP. Facing forward: Meeting the rehabilitation needs of cancer survivors. Oncology (Nurse Edition). 2010; 24(10):21-32. [PMID 21275322]

8. McEwen SE, Elmi S, Waldman M, Bishev M. Inpatient oncology rehabilitation in Toronto: A descriptive 18-month retrospective record review. Support Cancer Care. 201;20:1541-7. [PMID 21818640]

9. Nelson MB, Guo Y. A snapshot of inpatient cancer rehabilitation. Journal of Cancer Education. 2013; 28:676-8. [PMID 24037828]

10. Cheville AL, Beck LA, Peterson TL, Marks RS, Gamble GL. The detection and treatment of cancer-related functional problems in an outpatient setting. Support Cancer Care. 2008; 17:61-7. [PMID 18478275]

11. American Physical Therapy Association. Oncology PT Fact Sheets. 2015. Retrieved from: http://www.oncologypt.org/consumerresources/index.cfm

12. Longpré S, Newman R. The role of occupational therapy in oncology [Fact sheet]. Baltimore: American Occupational Therapy Association, 2011 Retrieved from: https://www.aota.org/Imedia/Corporate/Files/AboutOT/Professionals/WhatlsOT/RDP/Facts/Oncology\%20fact\%20sheet.pdf

13. American Speech Language Hearing Association. Swallowing problems (dysphagia) after head and neck cancers [Web page]. Traverse City: American Speech Language Hearing Association, 2015 Retrieved from: http://www.asha.org/public/speech/disorders/Swallowing-Problems-After-Head-and-Neck-Cancer/

14. Campbell C. Occupational therapy and cancer: Not just end of life care. OT Practice. 2010:15:19-21.

15. Thomas BC, Pandey M. Rehabilitation of the cancer patient. World Journal of Psycho-Social Oncology. 2012; 4:1-15.

16. National Comprehensive Cancer Network. NCCN guidelines version 2.2015: Definition of cancer-related fatigue. Fort Washington, PA: National Comprehensive Cancer Network, 2015 Retrieved from: http://www.nccn.org/professionals/physician gls/pdf/fatigue.pdf

17. Kim BR, Chun MH, Han EU, Kim DK. Fatigue assessment and rehabilitation outcomes in patients with brain tumors. Support Cancer Care. 2011; 20:805-12. [PMID 21533813]

18. Silver JK, Gilchrist LS. Cancer rehabilitation with a focus on evidence-based outpatient physical and occupational therapy interventions. American Journal of Physical Medicine \& Rehabilitation. 2011; 90(5):s5-15. [PMID 21765263]

19. Khan F, Amatya B. Factors associated with long-term functional outcomes, psychological sequelae and quality of life in persons after primary brain tumor. Journal of Neuro-Oncology. 2013; 111:355-66. [PMID 23292152]

20. Bartolo M, Zucchella C, Pace A, Lanzetta G, Vecchione C, Grillea G, Serrao M, Tassorelli C, Sandrini G, Pierelli F. Early rehabilitation after surgery improves functional outcome in inpatients with brain tumors. Journal of Neuro-Oncology. 2012; 107(3):537-44. [PMID 22124725]

21. Van Den Berg ME, Castellote JM, Mahillo-Fernandez, I, De Pedro-Cuesta J. Incidence of nontraumatic spinal cord injury: A Spanish cohort study (1972-2008). Archives of Physical Medicine and Rehabilitation. 2012; 93(2):325-31. [PMID 22289245]

22. Ruff RL, Adamson VW, Ruff SS, Wang X. Directed rehabilitation reduces pain and depression while increasing independence and satisfaction with life for patients with paraplegia due to epidural metastatic spinal cord compression. Journal of Rehabilitation Research \& Development. 2007; 44(1):1-10. [PMID 17551853]

23. Riesenberg H, Lubbe AS. Inpatient rehabilitation of lung cancer patients: A prospective study. Support Care Cancer. 2010; 18:877-22. [PMID 19714371]

24. Centers for Medicare and Medicaid Services. Medicare Benefit Policy Manual: Chapter 1- Inpatient hospital services covered under Part A. Baltimore, MD: Centers for Medicare and Medicaid Services, 2014 Retrieved from: https://www.cms.gov/Regulations-and-Guidance/Guidance/Manuals/ downloads/bp102c01.pdf 
25. Centers for Medicare and Medicaid Services. Inpatient Rehabilitation Facility Prospective Payment System. Baltimore,MD: Centers for Medicare and Medicaid Services, 2014 Retrieved from: http://www.cms.gov/Outreach-and-Education/MedicareLearning-Network-MLN/MLNProducts/downloads/ InpatRehabPaymtfctsht09-508.pdf

26. Mariotto AB, Yabroff KR, Shao Y, Feuer EJ, Brown ML. Projections of the cost of cancer in the United States: 2010-2020. Journal of the National Cancer Institute. 2011; 103(2):117-28. [PMID 21228314]

27. Mayo S, Booth E. Oncology rehabilitation program opens new doors to comprehensive recovery for cancer patients. Rehabilitation Quarterly: An Educational Journal of Baylor Institute for Rehabilitation. 2015; 2(1):1-3. 